\title{
Correlation between the expression of matrix metalloproteinase 9 and the expression of tissue inhibitor of metalloproteinase-1 of uterosacral ligament in uterine prolapse
}

\author{
Dhanny Primantara Johari Santoso, SpOG(K), M.Kes, Benny Hasan Purwara, SpOG(K), \\ Eppy Darmadi Achmad, SpOG(K), M.Kes
}

Department of Obstetrics and Gynaecology, Dr. Hasan Sadikin Hospital Bandung, Faculty of Medicine, Padjadjaran University, Bandung, Indonesia

\section{Objective}

This study aimed to analyze the correlation between the immunoexpression of matrix metalloproteinase 9 (MMP-9) and tissue inhibitor of metalloproteinase-1 (TiMP1) in the uterosacral ligaments in patients with uterine prolapse.

\section{Methods}

This analytic-correlative cross-sectional study included 32 patients who were admitted at the Hasan Sadikin General Hospital from July to December 2013. Sixteen of the patients had uterine prolapse, while the rest did not. The patients underwent total hysterectomy, radical hysterectomy, or staging laparotomy. MMP-9 and TiMP1 expression in the uterosacral ligaments was measured via immunohistochemical staining. The median expression per field of view was calculated using a histoscore.

Results

MMP-9 expression in patients with uterine prolapse was found to be higher than that in the control group. Meanwhile, TiMP1 expression showed no significant difference between the groups. Spearman's analysis showed a moderate correlation between the expression of MMP-9 and uterine prolapse incidence $(P=0.02)$, with a correlation coefficient of 0.574 .

\section{Conclusion}

There is a moderate correlation between MMP-9 expression and the incidence of uterine prolapse. It can be considered one of the primary etiologies of uterine prolapse.

Keywords: Matrix metalloproteinase 9; TiMP1; Correlation; Uterosacral ligament; Uterine prolapse

\section{Introduction}

Uterine prolapse generally results from poor cardinal or uterosacral ligament (USL) apical support, which allows downward protrusion of the cervix and uterus toward the introitus [1]. Incidence of uterine prolapse increases with age, and it usually occurs in post-menopausal women. It does not increase mortality, but does result in relatively high morbidity [2].

Uterine prolapse is associated with decreased quality of life, loss of productivity, and increased use of healthcare resources [3].
Received: 2021.04.19. Revised: 2021.08.15. Accepted: 2021.10.21. Corresponding author: Dhanny Primantara Johari Santoso, SpOG(K), M.Kes

Department of Obstetrics and Gynaecology, Dr. Hasan Sadikin Hospital Bandung, Faculty of Medicine, Padjadjaran University, Jl. Pasteur 38, Bandung 40161, Indonesia E-mail: santoso.dhanny99@gmail.com https://orcid.org/0000-0002-6481-2994

Articles published in Obstet Gynecol Sci are open-access, distributed under the terms of the Creative Commons Attribution Non-Commercial License (http://creativecommons. org/licenses/by-nc/3.0/) which permits unrestricted non-commercial use, distribution, and reproduction in any medium, provided the original work is properly cited.

Copyright (c) 2022 Korean Society of Obstetrics and Gynecology 


\section{Obstetrics \& Gynecology Science}

Dhanny Primantara Johari Santoso, et al. MMP-9 and TiMP1 USL in uterine prolapse

The incidence of uterine prolapse varies with race $[4,5]$. In a population-based study, data collection to assess the prevalence and incidence of uterine prolapse is complex due to the asymptomatic nature of early stage uterine prolapse (stage I and II). Patients with more severe cases (stage III or IV) generally visit the pelvic organ prolapse clinic.

In the USA, $11 \%$ of women aged up to 80 years undergo surgery for pelvic organ prolapse, and nearly one-third of the procedures are repeat surgeries [1]. Epidemiologic studies have shown that women have an $11-19 \%$ risk of undergoing operation for POP during their lifetime, and up to one third of them undergo additional operation for disease recurrence. Data from the Women's Health Initiative revealed anterior pelvic organ prolapse in $34.3 \%$, posterior wall prolapse in $18.6 \%$, and uterine prolapse in $14.3 \%$ of women included in the study [1].

However, the prevalence of uterine prolapse in Indonesia has not been comprehensively reported. During the last decade, a report from Hasan Sadikin Hospital in Bandung revealed an increase in the number of total hysterectomies, from 85 cases in 1999 to 121 cases in 2010, performed for uterine prolapse.

The pelvic organs are anatomically supported by the pelvic floor muscles and pelvic fascia consisting of connective tissue. The fascia is a loosely woven network of collagen, elastin, and fat, within which the blood vessels, lymphatic vessels, and nerves are present. Condensation of pelvic fascia in specific areas gives rise to ligaments [6].

The USL is the primary structure that supports a large part of the uterus [6]. The mechanical strength is maintained by type I and III collagen, constituting $70 \%$ of the extracellular matrix [7]. Uterine prolapse is clinically considered a consequence of decreased strength of the pelvic organ supporting tissue [4]. Prolapse occurs due to the alterations in collagen fiber content, which reduces the elasticity and durability of USL in supporting the anatomical integrity of the pelvic organs. Disorders of remodeling damaging type I and type III collagen fibers in the USL matrix after trauma may affect the mechanical function of the normal anatomic USL to support the pelvic organs $[4,8]$.

The balance between the synthesis and degradation of collagen is essential for maintaining tissue integrity and tensile strength during tissue remodeling. Degradation depends on a combination of matrix metalloproteinase (MMP) activity, regulation, release, and activation. Growth factor-binding proteins, cell surface receptors, and cell adhesion molecules also affect collagen degradation.

Tissue inhibitor of metalloproteinase-1 (TiMP1) is a protein that inhibits the metalloproteinase enzyme. TiMP1 plays a role in the activation of MMP-9. TiMP1 inhibits the active forms of MMP-2 and MMP-9. The role of collagen type III degradation in uterine prolapse remains controversial. Furthermore, the lack of remodeling of the extracellular matrix remains a question that cannot be ignored.

This study aimed to investigate the correlation between the expression of MMP-9 and TiMP1 in USL in patients with uterine prolapse. It is expected that the results of this study will provide a foundation for understanding the etiology of uterine prolapse.

\section{Materials and methods}

A cross-sectional design was used in this correlative analytic study that assessed the correlation between the expression of MMP-9 and the expression of TiMP1 in USL in uterine prolapse.

The population in this study consisted of patients admitted to Dr. Hasan Sadikin Hospital, Bandung, between July and December 2013, who had uterine prolapse, had not undergone operative treatment, and met the inclusion criteria. The inclusion criteria were as follows: patients who underwent hysterectomy for stage III and IV uterine prolapse according to the Pelvic Organ Prolapse-Quantification (POP-Q), had not received any prior hormonal therapy, and patients who were peri-menopausal or post-menopausal. The control group consisted of patients who underwent hysterectomy for indications other than uterine prolapse such as ovarian carcinoma, epithelial type cervical cancer stage I, endometrial carcinoma, ovarian cysts, or uterine myoma. The confounding factors were age, body mass index (BMI), number of vaginal deliveries, and parity. The inclusion criteria of perimenopausal and menopausal periods were carefully determined to suppress the effects of age.

The sample size was determined from the categorical correlative numerical analytic unpaired study of the two groups, which equaled to 16 patients in each group. Samples were collected by consecutive sampling.

An immunohistochemical examination of the MMP-9 antibody and TiMP1 obtained from the USL network was 


\title{
Obstetrics \& Gynecology Science
}

\author{
Vol. 65 , No. 1, 2022
}

performed after vaginal or abdominal hysterectomy. The USL network sample was processed by forming paraffin blocks stored in a freezer until it reached a specific temperature. These blocks were sliced to a thickness of 4 microns using a microtome.

Statistical analysis was used to determine the difference between the expression of MMP-9 and TiMP1 in the USL of the two groups. Statistical $P$-values were derived based on the Mann-Whitney analysis. Mann-Whitney analysis was selected because the data were not normally distributed. The strength of association between the two variables was investigated using Spearman's Rho analysis as the non-parametric correlation test. Statistical significance was determined based on the value of $P<0.05$.

\section{Results}

The samples in this study were derived from 32 paraffin blocks obtained from 16 patients with POP and 16 patients without POP (those with uterine myoma, benign ovarian tumors, malignant ovarian tumors, cervical cancer, or endometrial cancer). All patients with uterine prolapse underwent vaginal hysterectomy $(n=16)$.

Table 1 shows that the average age in the uterine prolapse group was higher than that in the control group. Older age was significantly associated with uterine prolapse $(P=0.0001)$. The majority of uterine prolapse patients had a normal BMI $\left(18.5-22.9 \mathrm{~kg} / \mathrm{m}^{2}\right)$. In contrast, the majority of patients in the control group were overweight (BMl, $23-24.9 \mathrm{~kg} / \mathrm{m}^{2}$ ). This means that a normal BMI was also significantly associated with uterine prolapse $(P=0.005)$. Patients with uterine prolapse also had a higher number of vaginal deliveries and parity $(P=0.001)$.

On the basis of the normality test, the values of both MMP9 and TiMP1 were non-normal. Therefore, the data are presented as median and interval values (minimal to maximal). The median MMP-9 expression in the uterine prolapse group was higher than that in the control group; however, the median TiMP expression was the same in both groups (Table 2). Statistical analysis using the Mann-Whitney test resulted in a $P$-value of 0.004 . This indicated that there was a significant difference in the level of MMP-9 expression between the two groups.

Table 3 shows the correlation between MMP-9 and TiMP1 expression calculated using the Spearman correlation, which showed a moderate correlation between the diagnoses. This correlation was negatively correlated $(r=-0.523, P=0.02)$, which means that the higher the diagnosis of uterine prolapse, the higher the MMP-9 score. Additionally, patient characteristics, age, BMI, parity, and number of vaginal deliveries were significantly different between the uterine pro-

Table 1. Characteristics of patients in the uterine prolapse and the control groups

\begin{tabular}{|c|c|c|c|c|c|}
\hline Characteristic & Uterine prolapse $(n=16)$ & Non-uterine prolapse $(n=16)$ & $P$-value & $\mathbf{t}$ & Z \\
\hline $\operatorname{Age}^{\mathrm{a})}$ & $62.56 \pm 6.5(51-59)$ & $53.63 \pm 2.73(49-73)$ & 0.0001 & 5.070 & \\
\hline Body mass index ${ }^{b}$ & $21.35(20-26.84)$ & $23.87(20-29.28)$ & 0.005 & & 2.829 \\
\hline Parity & $6.06 \pm 2.48$ & $3.56 \pm 1.31$ & 0.001 & 3.552 & \\
\hline Total vaginal labor ${ }^{a)}$ & $5.19 \pm 1.83$ & $3.25 \pm 1.12$ & 0.001 & 3.602 & \\
\hline
\end{tabular}

Values are presented as mean \pm standard deviation (min-max) or median (interval).

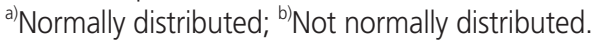

Table 2. Comparison of histoscore of MMP-9 and TiMP1 expression between the uterine prolapse and the control groups

\begin{tabular}{|c|c|c|c|c|}
\hline \multirow{2}{*}{ Variable } & \multicolumn{2}{|c|}{ Group research } & \multirow{2}{*}{$P$-value } & \multirow{2}{*}{ Z } \\
\hline & Uterine prolapse $(n=16)$ & Non-uterine prolapse $(n=16)$ & & \\
\hline MMP-9 & $6.00(2-12)$ & $2.50(2-12)$ & $0.004^{\text {a) }}$ & -2.912 \\
\hline TiMP1 & $0(0-2)$ & $0(0-12)$ & 0.619 & -0.498 \\
\hline
\end{tabular}

Values are presented as median (interval).

MMP-9, matrix metalloproteinase 9; TiMP1, tissue inhibitor of metalloproteinase-1.

a) Significant difference. 


\section{Obstetrics \& Gynecology Science}

Dhanny Primantara Johari Santoso, et al. MMP-9 and TiMP1 USL in uterine prolapse

lapse and the control groups. Therefore, it was necessary to perform a linear regression for these $5 X$ variables (diagnosis, age, BMI, parity, and vaginal delivery) to ascertain whether these characteristics affected the MMP-9 score.

Table 4 shows that the results of the linear regression were significant $(P=0.030, r=0.603)$. However, only the diagnostic variable (i.e., presence of uterine prolapse) was significantly associated with MMP-9 expression $(P=0.017)$. Since only one variable showed a significant relationship, multivariate regression was not needed. To ensure that age, BMI, parity,

Table 3. Correlation between the expression of MMP-9 and TiMP1 in the uterine prolapse and the control groups

\begin{tabular}{lcc}
\hline & $\begin{array}{c}\text { Correlation } \\
\text { coefficieny } \mathbf{r}\end{array}$ & $\begin{array}{c}\text { Significancy } \\
\boldsymbol{P} \text {-value }\end{array}$ \\
\hline Uterine prolapse & $0.523^{\mathrm{a})}$ & $0.02^{\mathrm{b})}$ \\
Non-uterine prolapse & $0.362^{\mathrm{a})}$ & 0.168 \\
\hline
\end{tabular}

MMP-9, matrix metalloproteinase 9; TiMP1, tissue inhibitor of metalloproteinase-1.

${ }^{\text {a) }}$ Moderate correlation; ${ }^{\text {b) }}$ Significant difference. and delivery variables did not affect the TiMP1 score, linear regression was performed for the uterine prolapse and the control groups.

Table 5 shows the linear regression analysis to determine the effect of patient characteristics on uterine prolapse. It can be seen that none of the characteristics met the requirements for multivariate analysis, and therefore, it was not performed $(P=0.51, r=0.488)$.

\section{Discussion}

The characteristics of the patients in this study included age, $\mathrm{BMI}$, parity, and the number of vaginal deliveries. These characteristics act as risk factors for uterine prolapse [4] and potentially confounding variables that may affect the study's validity.

A wide range of age among the 32 patients with and without uterine prolapse can also lead to bias. Therefore, age homogeneity was difficult to achieve in this study. The youngest

Table 4. Simple linear regression analysis for determining the association between patient characteristics (age, BMI, vaginal delivery, and parity) and MMP-9 expression in the uterine prolapse group

\begin{tabular}{lcccccc}
\hline Model & $\begin{array}{c}\text { Unstandardized } \\
\text { coefficients }\end{array}$ & $\begin{array}{c}\text { Standard error } \\
\text { coefficient B }\end{array}$ & $\begin{array}{c}\text { Standardized } \\
\text { coefficients }\end{array}$ & $\mathbf{t}$ & $\boldsymbol{P}$-value & $\begin{array}{c}\text { Regression } \\
\text { Linear regres- } \\
\text { sion } \boldsymbol{P} \text {-value }\end{array}$ \\
\hline Constant & 29.665 & 9.753 & Beta & 3.042 & 0.005 & 0.603 \\
Diagnosis & -5.211 & 2.034 & -0.711 & -2.562 & 0.017 & 0.03 \\
Age & -0.208 & 0.128 & -0.374 & -1.629 & 0.115 & \\
BMl & -0.184 & 0.354 & -0.101 & -0.519 & 0.608 \\
Parity & -0.224 & 0.595 & -0.141 & -0.377 & 0.709 \\
Total vaginal & 0.325 & 0.778 & 0.157 & 0.418 & 0.679 \\
labor & & & & & & \\
\hline
\end{tabular}

BMI, body mass index; MMP-9, matrix metalloproteinase 9.

Table 5. Simple linear regression analysis to determine the association between patient characteristics (age, BMI, vaginal delivery, and parity) and uterine prolapse

\begin{tabular}{lcccccc}
\hline Model & $\begin{array}{c}\text { Unstandardized } \\
\text { coefficients }\end{array}$ & $\begin{array}{c}\text { Standar error } \\
\text { coefficient B }\end{array}$ & $\begin{array}{c}\text { Standardized } \\
\text { coefficients }\end{array}$ & $\mathbf{t}$ & $\boldsymbol{P}$-value & $\begin{array}{c}\text { Regression } \\
\text { Linear regres- } \\
\text { sion } \boldsymbol{P} \text {-value }\end{array}$ \\
\hline Constant & 29.665 & 9.753 & Beta & 3.042 & 0.005 & 0.488 \\
Age & -0.208 & 0.128 & -0.374 & -1.629 & 0.115 & 0.51 \\
BMl & -0.184 & 0.354 & -0.101 & -0.519 & 0.608 & \\
Parity & -0.224 & 0.595 & -0.141 & -0.377 & 0.709 \\
Total vaginal & 0.325 & 0.778 & 0.157 & 0.418 & 0.679 \\
$\quad$ labor & & & & & & \\
\hline
\end{tabular}

BMI, body mass index. 


\section{Obstetrics \& Gynecology Science}

Vol. 65 , No. 1, 2022

patient was 49 years old, and the oldest was 73 years old. Age is a risk factor for POP, which can affect the validity of the research results.

Age is closely associated with menopause, which directly affects collagen synthesis. Low estrogen receptors make MMP-9 associated with increased flexural strength of the USL [8]. Although postmenopausal women have been reported to have a higher risk of uterine prolapse than younger women, factors such as the number of parity and a high BMI also increase the risk of uterine prolapse $[5,9]$. Although $B M I$ was not significantly correlated with POP stage $(P=0.271)$ [10].

In this study, patients in the uterine prolapse group had more vaginal deliveries $(P=0.001)$. According to Strinic et al. [11], uterine prolapse is more common, especially in women who gave birth vaginally more than thrice or gave birth to a macrosomic baby.

The median MMP-9 expression in the uterine prolapse group was higher than that in the control group. The results of the analysis of MMP-9 expression (Table 2) in this study are consistent with the findings of a previous research conducted by Moalli et al. [12]. They found an increase in MMP-9 expression in patients with uterine prolapse [12]. Our results are also consistent with previous studies showing that the increased expression levels of MMP-9 and the reduced expression levels of TiMP1 are directly associated with the presence of uterine prolapse by Wang et al. [13]. This indicates that the differential expression levels of MMP-9 and TiMP1 are correlated with the occurrence and development of uterine prolapse.

The excessive state of degradation is also regulated by endogenous inhibitors, as well as by TiMP1. The reduction in TiMP1 levels in the uterine prolapse group (Table 2) is also consistent with that reported by Chen et al. [14].

Based on these results, it can be assumed that the decrease in the USL tensile strength and flexibility in uterine prolapse in the patients of this study was related to the state of excess degradation.

These findings demonstrate that the differential expression of MMPs and TiMPs is involved in the onset and development of uterine prolapse. A practical method to treat uterine prolapse may be to restrict MMP-9 expression and increase TiMP expression levels. Therefore, decreasing collagen degradation and increasing the level of the local binding protein, thus enhancing the tissue elasticity of the uterus.
Nevertheless, this research has several limitations. First, the sample size was relatively small. The second limitation concerns the variability of cases, since, ideally, multiple stages of uterine prolapse should be included in the study. This was not possible because of the unavailability of patients with stage I or II prolapse for sampling.

\section{Conflict of interest}

The authors declare that they have no conflicts of interest.

\section{Ethical approval}

The Research Ethics Committees of both Universitas Padjajaran and Dr. Hasan Sadikin Hospital, Bandung approved this study.

\section{Patient consent}

Informed consent was not required because this was a retrospective study.

\section{Funding information}

None.

\section{Acknowledgments}

The authors thank the research team at the Department of Obstetrics and Gynecology, Faculty of Medicine, Universitas Padjadjaran/Dr. Hasan Sadikin Hospital, Bandung, for coordinating the study and data analysis activities.

\section{References}

1. Jeon MJ. Surgical decision making for symptomatic pelvic organ prolapse: evidence-based approach. Obstet Gynecol Sci 2019;62:307-12.

2. MacLennan AH, Taylor AW, Wilson DH, Wilson D. The 


\section{Obstetrics \& Gynecology Science}

Dhanny Primantara Johari Santoso, et al. MMP-9 and TiMP1 USL in uterine prolapse

prevalence of pelvic floor disorders and their relationship to gender, age, parity and mode of delivery. BJOG 2000;107:1460-70.

3. Awwad J, Sayegh R, Yeretzian J, Deeb ME. Prevalence, risk factors, and predictors of pelvic organ prolapse: a community-based study. Menopause 2012;19:1235-41.

4. Mant J, Painter R, Vessey M. Epidemiology of genital prolapse: observations from the oxford family planning association study. Br J Obstet Gynaecol 1997;104:57985.

5. Bump RC, Norton PA. Epidemiology and natural history of pelvic floor dysfunction. Obstet Gynecol Clin North Am 1998;25:723-46.

6. Sabbagh MD, Manrique O, Ciudad P, Adabi KBA, Huang $\mathrm{TCT}$, Chen HC, et al. Abstract: gender reassignment surgery using the pedicle transverse colon flap for vaginal reconstruction: a clinical outcome and sexual function evaluation study. Plast Reconstr Surg - Glob Open 2018;6(8 Suppl):138-9.

7. Bureau M, Carlson KV. Pelvic organ prolapse: a primer for urologists. Can Urol Assoc J 2017;11(6Suppl2):S125-30.

8. Ewies AA, Al-Azzawi F, Thompson J. Changes in extracellular matrix proteins in the cardinal ligaments of post-menopausal women with or without prolapse: a computerized immunohistomorphometric analysis. Hum
Reprod 2003;18:2189-95.

9. Schaffer JI, Wai CY, Boreham MK. Etiology of pelvic organ prolapse. Clin Obstet Gynecol 2005;48:639-47.

10. Kim BH, Lee SB, Na ED, Kim HC. Correlation between obesity and pelvic organ prolapse in Korean women. Obstet Gynecol Sci 2020;63:719-25.

11. Strinic T, Vulic M, Tomic S, Capkun V, Stipic I, Alujevic I. Increased expression of matrix metalloproteinase-1 in uterosacral ligament tissue from women with pelvic organ prolapse. Acta Obstet Gynecol Scand 2010;89:8324.

12. Moalli PA, Talarico LC, Sung VW, Klingensmith WL, Shand SH, Meyn LA, et al. Impact of menopause on collagen subtypes in the arcus tendineous fasciae pelvis. Am J Obstet Gynecol 2004;190:620-7.

13. Wang X, Li Y, Chen J, Guo X, Guan H, Li C. Differential expression profiling of matrix metalloproteinases and tissue inhibitors of metalloproteinases in females with or without pelvic organ prolapse. Mol Med Rep 2014; 10:2004-8.

14. Chen BH, Wen Y, Li H, Polan ML. Collagen metabolism and turnover in women with stress urinary incontinence and pelvic prolapse. Int Urogynecol J Pelvic Floor Dysfunct 2002;13:80-7; discussion 87. 